\title{
PARÂMETROS ELETROCARDIOGRÁFICOS DE CASCAVÉIS (Crotalus durissus, Linnaeus, 1758) EM CATIVEIRO
}

\author{
Daniel Rodrigues Stuginski ${ }^{1}$, Wilson Fernandes ${ }^{1}$, Kathleen Fernandes Grego ${ }^{1}$ \\ 1 Instituto Butantan
}

Endereço para correspondência: Daniel Rodrigues Stuginski: dstuginski@yahoo.com.br

\begin{abstract}
RESUMO: Apesar de a eletrocardiografia ser vastamente empregada na clínica veterinária, esta ferramenta ainda é pouco utilizada no diagnóstico de patologias em serpentes. Este trabalho teve como objetivo a caracterização dos parâmetros eletrocardiográficos da cascavel Crotalus durissus em cativeiro, sob condições controladas. Para isto, 23 serpentes foram submetidas ao exame eletrocardiográfico, aonde os seguintes parâmetros foram analisados: a frequência cardíaca, ritmo, eixo cardíaco, ondas $\mathrm{P}$ e T, complexo QRS, assim como os intervalos P-R e segmento S-T nas derivações cardíacas DI, DII, DIII, aVF aVL e aVR. Os resultados obtidos demonstraram que a técnica foi eficiente nas mensurações das deflexões cardíacas, porém a análise da frequência e ritmo geralmente foram prejudicados devido a um viés estresse-induzido e sua interpretação deve ser cautelosa. Os parâmetros eletrocardiográficos de Crotalus durissus determinados neste trabalho foram bastante consistentes e com mínimas variações, o que parece possibilitar sua utilização no diagnóstico das doenças cardíacas em serpentes. A escassez de padrões eletrocardiográficos em serpentes torna muito difícil a comparação e a interpretação de como as variáveis morfológicas, fisiológicas e ecológicas podem influenciar os parâmetros eletrocardiográficos, por este motivo, novas pesquisas que determinem os padrões eletrocardiográficos de diferentes espécies serão absolutamente necessárias para o uso do eletrocardiograma como ferramenta diagnóstica na rotina clínica.
\end{abstract}

Palavras-chave: répteis; serpentes; eletrocardiograma.

\section{ELECTROCARDIGRAPHIC PARAMETERS OF RATTLESNAKES (CROTALUS DURISSUS, LINNAEUS, 1758 ) IN CAPTIVITY}

\begin{abstract}
Although electrocardiography is widely used in veterinary practice, this tool still has little application in diagnosing diseases of snakes. This study aimed to characterize the electrocardiographic parameters of Crotalus durissus in captivity, under controlled conditions. For this, 23 snakes were subjected to ECG examination, where the following parameters were analyzed: heart rate, rhythm, cardiac axis, $\mathrm{P}$ and T waves, QRS complex, as well as the P-R and S-T intervals in the heart leads DI, DII, DIII, aVF, aVL and aVR The results showed that the technique used was effective to the measurements of cardiac deflections, but the analysis of heart rate and rhythm is generally impaired by a bias-induced stress and its interpretation should be cautious. The electrocardiographic parameters of Crotalus durissus determined in this study were fairly consistent, with minor variations, which seem to allow its use for cardiac disease diagnosis in snakes. The scarcity of electrocardiographic patterns for snakes makes difficult a comparison and interpretation of how the morphological, physiological and ecological data can influence the electrocardiogram, therefore, further research to determine the electrocardiographic pattern of different species will be absolutely necessary to the use of the electrocardiogram as a diagnostic tool in clinical routine.
\end{abstract}

Key Words: reptiles; snakes; electrocardiography. 


\section{INTRODUÇÃO}

O eletrocardiograma é um exame amplamente utilizado em medicina veterinária de pequenos animais (Kittleson e Kienle, 1998; Claude et al., 2010; Kijtawornra et al., 2010), e seu uso na medicina de répteis tem crescido ao longo dos anos (Jacobson et al., 1991; Kik e Mitchell, 2005). O mecanismo básico de ação do eletrocardiograma consiste na impressão gráfica do sentido, direção e intensidade dos vetores elétricos cardíacos em relação a determinados pontos fixos (eletrodos) (Tilley $\mathrm{e}$ Goodwin, 2001). Pelo estudo eletrocardiográfico podemos determinar a frequência, o ritmo cardíaco, o tempo e o sentido de despolarização e repolarização de cada uma das câmaras cardíacas, o cálculo do eixo cardíaco, entre outras variáveis que podem ocorrer em cada deflexão do registro (Tilley e Goodwin, 2001), tornando-o assim uma incrível ferramenta para estudos fisiológicos ou para a determinação diagnóstica (Billman, 2006; Dennis et al., 2007).

Os parâmetros eletrocardiográficos para o ser humano e para os animais domésticos já estão bem determinados, porém, infelizmente ainda são escassos em répteis (Davies et al., 1951; Kaplan e Schwartz, 1963; Jayasinghe e Fernando, 1964; Valentinuzi et al., 1969a,b; Cook e Westron, 1979; Heaton et al., 1994) sendo ainda mais raros em serpentes (Mullen, 1967).

Sabidamente as serpentes, tais quais os demais répteis, sofrem uma enorme influência de fatores abióticos (p. ex. temperatura) e bióticos (p. ex status prandial) sobre seu metabolismo (Risher e Claussen, 1987; Beaupre, 1995; Secor, 2009), o que pode influenciar seu desempenho cardíaco de maneira diversa, apesar disto tal fato foi pouco relevado nos estudos dos parâmetros eletrocardiográficos destes animais (McDonald e Heath, 1971, Risher e Claussen, 1987; Liu e Li, 2005). Além disso, a grande diversidade de hábitos, morfologia ( $p$. ex. posicionamento cardíaco) (Badeer, 1998; Gartner et al., 2010) dificulta algumas generalizações dos padrões eletrocardiográficos, 0 que torna especialmente relevante 0 estudo das diferentes espécies separadamente.

Neste presente trabalho utilizamos 23 serpentes da espécie Crotalus durissus como modelo experimental de um estudo eletrocardiográfico espécieespecífico e controlado (estado prandial conhecido e temperatura estável) na tentativa da determinação de alguns dos parâmetros eletrocardiográficos destes animais.

\section{MATERIAL E MÉTODOS}

Animais: Para o estudo foram utilizados 23 animais da espécie Crotalus durissus procedentes do laboratório de Herpetologia do Instituto Butantan, de ambos os sexos (12 machos e 11 fêmeas), cujo peso variou entre $1089 \pm 212 \mathrm{~g}$, aparentemente saudáveis (animais sem histórico de doenças durante no mínimo 3 anos de cativeiro).

Os animais foram mantidos durante 0 experimento em caixas de madeira, medindo $53 \times 37 \times 25 \mathrm{~cm}$, forradas com papelão corrugado e água ad libitum. A alimentação a estes animais foi fornecida a cada 30 dias, sendo composta de Rattus novergicus procedentes do biotério geral do Instituto Butantan.

Procedimento eletrocardiográfico: Durante a realização do exame, os animais foram contidos com um tubo plástico transparente com $10 \mathrm{~cm}$ de diâmetro. O coração foi então localizado pela palpação e observação do choque precordial. O exame foi realizado com o aparelho de eletrocardiograma (Info Fumbec) e rodado em papel 
convencional de eletrocardiografia, os eletrodos usados foram do tipo jacaré padrão e presos diretamente sobre a pele do animal, sendo os eletrodos craniais dispostos um de cada lado a 8 $\mathrm{cm}$ craniais ao coração do animal (eletrodo vermelho no lado direito e eletrodo amarelo no lado esquerdo) e os outros eletrodos foram colocados um de cada lado a $8 \mathrm{~cm}$ caudais ao coração (eletrodo preto do lado direito e eletrodo verde do lado esquerdo. Durante o procedimento os animais foram mantidos em decúbito ventro-dorsal. Os eletrodos, bem como a porção da pele em contato com os eletrodos foram embebidos em álcool-gel para possibilitar maior condutividade entre os eletrodos e o animal.

Todos os animais foram submetidos a um jejum de 25 dias antes da eletrocardiografia e a temperatura ambiente em que estes animais foram mantidos durante o experimento foi de $26,5 \pm 1,4^{\circ} \mathrm{C}$, com umidade relativa de $65-75 \%$. Foram analisadas as deflexões referentes às ondas $\mathrm{P}, \mathrm{T}$, complexo QRS, intervalo P-R e segmento S-T, sob as derivações DI, DII, DIII, aVR, aVL e aVF (Figura 1).

Para a análise estatística foi utilizado o programa Instat5 (Teste-T student) e o nível de significância para todos os achados foi o de $\mathrm{P}<0,05$.

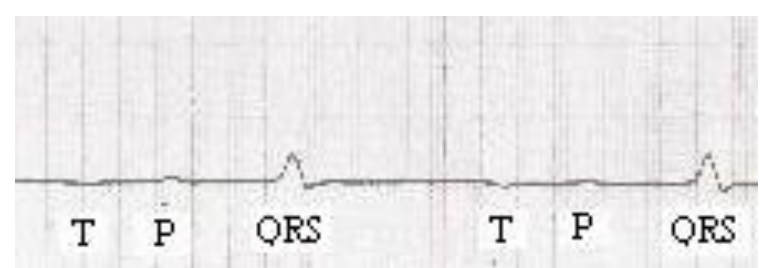

Figura 1- Impressão eletrocardiográfica de Crotalus durissus em DII, $50 \mathrm{~mm} / \mathrm{s}$ e sensibilidade $\mathrm{N}$, demonstrando as ondas $\mathrm{P}, \mathrm{T}$ e o complexo QRS.

\section{RESULTADOS}

O peso dos animais não variou significativamente entre os diferentes sexos $(P>0,05)$. A frequência cardíaca mensurada pelo eletrocardiograma foi de 45,38 $\pm 4,51 \mathrm{bpm}$, sendo que também não houve diferença significativa entre a frequência cardíaca de machos e fêmeas $(P>0,05)$. Com relação ao ritmo, todas as serpentes eletrocardiografadas,

independentemente do sexo, apresentaram um ritmo sinusal normal com o intervalo $R-R$ variando menos de $5 \%$, não sendo detectada nenhuma influência da respiração do animal sobre o ritmo cardíaco. O eixo cardíaco variou da seguinte forma: $64 \%$ dos eixos entre 60 e 90 graus, $12 \%$ entre 30 e 60 graus e $24 \%$ em 60 graus.

Nenhuma diferença estatisticamente significativa entre machos e fêmeas foi detectada nas medições de ondas ou intervalos $(P>0,05)$. As tabelas 1-3 e a figura 2 apresentam os resultados das deflexões eletrocardiográficas encontradas e os respectivos intervalos, bem como as polaridades do complexo QRS e a porcentagem de detecção das ondas $\mathrm{P}$ e $\mathrm{T}$.

Tabela 1 - Polaridade do complexo QRS nas diferentes derivações.

\begin{tabular}{lllllll}
\hline Derivação & DI & DII & DIII & aVR & aVL & aVF \\
\hline Positivo & $100 \%$ & $100 \%$ & $100 \%$ & $0 \%$ & $12 \%$ & $100 \%$ \\
Negativo & $0 \%$ & $0 \%$ & $0 \%$ & $100 \%$ & $64 \%$ & $0 \%$ \\
Isoelétrico & $0 \%$ & $0 \%$ & $0 \%$ & $0 \%$ & $24 \%$ & $0 \%$ \\
\hline
\end{tabular}


Tabela 2 - Duração e amplitude das ondas e intervalos em DII.

\begin{tabular}{|c|c|c|c|c|c|c|c|c|}
\hline Onda & $\begin{array}{l}P \\
\text { (s) }\end{array}$ & $\begin{array}{l}P \\
(\mathrm{mV})\end{array}$ & $\begin{array}{l}\text { P-R } \\
\text { (s) }\end{array}$ & $\begin{array}{l}\text { QRS } \\
\text { (s) }\end{array}$ & $\begin{array}{l}\text { QRS } \\
(\mathrm{mV})\end{array}$ & $\begin{array}{l}\text { S-T } \\
\text { (s) }\end{array}$ & $\begin{array}{l}\mathrm{T} \\
\text { (s) }\end{array}$ & $\begin{array}{l}\mathrm{T} \\
(\mathrm{mV})\end{array}$ \\
\hline Média $\quad \pm$ & 0,05 & 0,06 & 0,33 & 0,12 & 0,38 & 0,40 & 0,21 & 0,07 \\
\hline \multirow[t]{2}{*}{ desv.p. } & \pm & \pm & \pm & \pm & \pm & \pm & \pm & \pm \\
\hline & 0,01 & 0,02 & 0,04 & 0,01 & 0,09 & 0,07 & 0,04 & 0,05 \\
\hline \multirow[t]{2}{*}{ Intervalo } & $0,02-$ & $0,04-$ & $0,24-$ & $0,06-$ & $0,2-$ & $0,24-$ & $0,18-$ & $0,05-$ \\
\hline & $0,08 \mathrm{~s}$ & 0,01 & $0,42 \mathrm{~s}$ & 0,14 & 0,6 & 0,5 & 0,3 & 0,2 \\
\hline $\begin{array}{l}\text { Percentual } \\
\text { de }\end{array}$ & $62 \%$ & $62 \%$ & $62 \%$ & $96 \%$ & $96 \%$ & $45 \%$ & $45 \%$ & $45 \%$ \\
\hline detecção & & & & & & & & \\
\hline
\end{tabular}

Tabela 3- Porcentagens de detecção das ondas $\mathrm{P}$ e T nas diferentes derivações.

\begin{tabular}{llllll}
\hline Ondas & DI & DIII & aVR & aVL & aVF \\
\hline $\mathrm{P}$ & $6,7 \%$ & $56 \%$ & $30 \%$ & $13,5 \%$ & $13,5 \%$ \\
$\mathrm{~T}$ & $15 \%$ & $40 \%$ & $33 \%$ & $2,5 \%$ & $2 \%$ \\
\hline
\end{tabular}

Figura 2- Duração do complexo QRS, intervalos $\mathrm{P}-\mathrm{R}$ e segmento S-T em relação ao intervalo $\mathrm{R}-\mathrm{R}$.

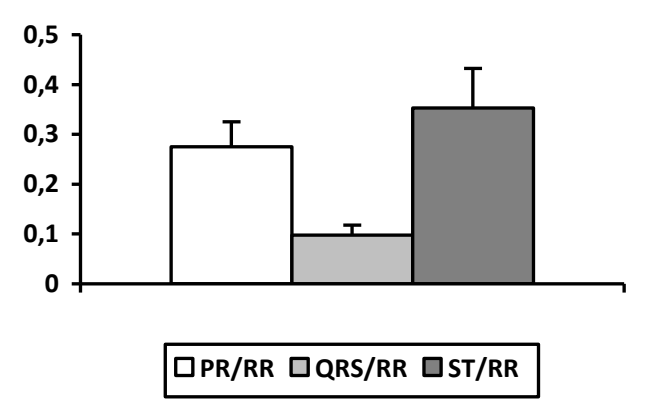

\section{DISCUSSÃO}

O uso de eletrodos tipo jacaré posicionados diretamente sobre a pele do animal (Cruz e Junkes, 2008; Shoemaker e Zandvliet, 2005) poupa trabalho ao clínico que deseja fazer uma análise rápida do perfil eletrocardiográfico do animal, embora técnicas diferentes e igualmente práticas como o uso de eletrodos com colocação de agulha sub-dérmica, tenham igualmente sido empregadas com sucesso para répteis (Liu e $\mathrm{Li}$, 2005; Shoemaker e Zandvliet, 2005).
Técnicas diferentes, como o uso de data loggers foram aplicadas com sucesso para a determinação de certos parâmetros cardíacos (Campbell et al., 2006).

O uso de data logger possibilita, por exemplo, uma aferição precisa da frequência e ritmo cardíaco do animal em descanso, já que após a introdução do aparelho via cirurgia o animal é deixado em recuperação e seus parâmetros cardíacos são então aferidos com uma menor influência do fator estressante da contenção (Cruz e Junkes, 2008), todavia, seu alto custo e a necessidade de realizar uma pequena cirurgia o tornam pouco viável na rotina clínica. O posicionamento do animal em decúbito dorso-ventral parece ser especialmente importante para um registro acurado da eletrocardiografia de serpentes, já que para animais de corpo cilíndrico, outras posições podem potencialmente influenciar na determinação dos vetores cardíacos (Shoemaker e Zandvliet, 2005).

O uso de anestésicos durante o exame eletrocardiográfico, apesar de muitas vezes necessário, pode levar a alterações no registro ( $\mathrm{Holz}$ e $\mathrm{Holz}$, 1995; Shoemaker e Zandvliet, 2005), a contenção com o tubo plástico, provou ser útil, pois possibilitou um exame sem as interferências causadas pela contração muscular e sem o viés da aferição dos parâmetros durante uma contenção química.

Com relação à frequência cardíaca, apesar de certa congruência com as frequências previamente obtidas com o eletrocardiograma (Mullen, 1967; Shoemaker e Zandvliet, 2005), os resultados apresentam um viés induzido pela contenção dos animais (Cruz e Junkes, 2008), sendo assim a frequência cardíaca relativamente alta detectada em nosso trabalho, quando comparada aos dados obtidos através do data logger na mesma espécie de cascavel (Campbell et al., 2006), 
refletem possivelmente uma taquicardia reflexa induzida pelo estresse da contenção (Cruz e Junkes, 2008).

A notória ritimicidade detectada em nossos traçados, também foi notada em um espécime de python por Shoemaker e Zandvliet (2005), todavia, este ritmo sinusal normal pode ser um reflexo do aumento da frequência cardíaca causada pelo estresse da contenção, já que a influência do ciclo respiratório no ritmo cardíaco (arritimia sinusal respiratória) foi notada em análises realizadas sem o componente estressante da contenção, sob frequências cardíacas menores (Campbell et al., 2006).

A detecção da onda SV nos eletrocardiogramas de répteis parece variar conforme a espécie e a derivação utilizada na leitura, sendo esta onda referente a despolarização do seio venoso do coração (Cook e Westrom, 1979; Terrel et al., 1994). A presença de ondas SV parece ser muito mais notória em leituras pré-cordiais (Terrel et al., 1994), porém, por não terem sido realizadas neste trabalho tais leituras, a ausência de onda SV no padrão eletrocardiográfico de Crotalus durissus deve ser vista com cautela

Os intervalos P-R e complexos QRS determinados por Mullen (1967) diferem um pouco de nossos achados, principalmente quanto às médias, o que pode ser explicado pelo uso de um pool de espécies, diferentemente de nosso trabalho que utilizou uma determinação espécie-específica e sob condições térmicas controladas. Vale à pena ressaltar que no trabalho de Mullen (1967) foram também utilizadas espécies de Crotalus (C. cerastes, C. scutulatus e $\mathrm{C}$. viridis) que normalmente possuem um tamanho inferior às $C$. durissus (Campbell e Lamar 2004), todavia, por não constar a massa destes animais no trabalho, uma análise mais precisa para os achados fica restrita.
Os dados desse estudo corroboram os de Hagensen et al. (2008) para animais em atividade com relação a razão $P R / R R$ para a espécie, e conforme 0 esperado a relação QRS/RR nesse trabalho foi mais alta do que a achada no trabalho supracitado, já que a frequência cardíaca dos nossos animais foi mais elevada e com isso a relação de QRS/RR tende a aumentar (Hagensen et al., 2008).

O segmento S-T e a onda $T$ obtiveram moderada representatividade na leitura DII, concordando plenamente com os resultados de Mullen (1967) no tocante à dificuldade de se calcular a amplitude de $\mathrm{T}$, apesar disso, a duração das ondas $T$ em ambos os trabalhos é virtualmente igual, e a relação ST/RR foi muito próxima às detectadas anteriormente para a espécie (Hagensen et al., 2008), confirmando a independência deste intervalo da frequência.

\section{CONCLUSÃO}

A eletrocardiografia em répteis representa uma interessante ferramenta para estudos diagnósticos e fisiológicos em serpentes, sendo especialmente interessante para avaliações cardíacas baseadas na leitura das ondas $P, T$ e complexo QRS. A avaliação de frequência e ritmo deve ser feita com cautela já que o procedimento apresenta uma clara influência nestes parâmetros quando comparada a outros métodos. Além disso, é necessária a obtenção de padrões espécieespecíficos e sob as mesmas condições, já que estes parâmetros variam consistentemente dentre as diferentes espécies e sob diferentes condições (p. ex. temperatura). 


\section{REFERÊNCIAS}

BADEER, H.S. Anatomical position of heart in snakes with vertical orientation: a new hypothesis. Comparative Biochemistry and Physiology Part A Molecular \& Integrative Physiology, v.119A, n.1, p.403-405, 1998.

BEAUPRE, S.J. Effects of geographically variable thermal environment on bioenergetics of mottled rock rattlesnakes. Ecology, v.76, n.5, p.1655-1665, 1995.

BILLMAN, G.E. A comprehensive review and analysis of 25 years of data from an in vivo canine model of sudden cardiac death: Implications for future anti-arrhythmic drug development. Pharmacology and Therapeutics, v.111, n.3, p.808-835, 2006.

CAMPBELL, H.A.; LEITE, C.A.C.; WANG, T.; SKALS, M.; ABE, A.S.; EGGINTON, S.; RANTIN, F. T.; BISHOP, C.M.; TAYLOR, E.W. Evidence for a respiratory component, similar to mammalian respiratory sinus arrythmia, in heart rate variability signal from rattlesnake, Crotalus durissus terrificus. The Journal of Experimental Biology, v.209, p.2628-2636, 2006.

CAMPBELL, J.A.; LAMAR, W.W. The Venomous Reptiles of the Western Hemisphere,Cornell University Press, 2004, v.2, Cap. 1, p. 477-597.

CLAUDE, A.K.; RIEDESEL, D.H.; RIEDESEL E.A. Electrocardiography-guided and retrospective analysis of central venous catheter placement in the dog, Veterinary Anaesthesia and Analgesia, v.37, n.2, p.97-105, 2010.

COOK, R.A.; WESTROM, W. Cardiac anatomy, cardiac physiology and eletrocardiology of reptiles. American Association of Zoo Veterinarians Annual proceedings, v.1979, p.16-22, 1979.

CRUZ, J.G.P.; JUNKES, L. Controle autonômico da frequência cardíaca em Spilotes pullatus (Colubridae) como determinante da resposta de medo. Arquivo Brasileiro de Medicina Veterinária e Zootecnia, v.60, n.6, p.14681471, 2008.

DAVIES, F. E.; Francis, T.B.; KING, T.S. Eletrocardiogram of the crocodilian heart. Nature, v.167, p.146, 1951.

DENNIS, S.G.; WOTTON, P.R.; BOSWOOD, A.; FLAHERTY, D. Comparison of the effects of thiopentone and propofol on the electrocardiogram of dogs. Veterinary Record, v.160, n.20, p.681-686, 2007.
GARTNER, G.E.A.; HICKS, J.W.; MANZANI, P.R.; ANDRADE, D.V.; ABE, A.S.;WANG, T.; SECOR, S.M.; GARLAND, T. Phylogeny, ecology and heart position in snakes. Physiological and Biochemical Zoology, v.83, n.1, p. 43-54, 2010.

HAGENSEN, M.K.; ABE, A.S.; ERLING, F.; WANG, T. Physiological importance of the coronary arterial blood supply to the rattlesnake heart. The journal of experimental biology, v.211, p.3588-3593, 2008.

HEATON, J.; TERELL, G.; KING, R.R. Characterization of the electrocardiogram of American alligator (Alligator mississippiensis). Journal of Zoo and Wildlife Medicine, v.25, n.1, p.40-47, 1994.

HOLZ, R.M.; HOLZ, P. Electrocardiography in anesthetised red-eared sliders (Trachemys scripta elegans), Research in Veterinary Science, v.58, p.67-69, 1995.

JACOBSON, E.R.; HOMER, B.; ADAMS, W. Endocarditis and congestive heart failure in a Burmese Python (Python molurus bivitatus), Journal of Zoo and Wildlife Medicine, v.22, n.2, p.245-248, 1991.

JAYASINGHE, J.B.; FERNANDO, S. D. A.Some reptilians electrocardiograms. Ceylon Veterinary Journal, v. 12, p.43-16, 1964.

KAPLAN, H.M.; SCHWARTZ, C. Eletrocardiography in turtles. Life Sciences, v.9, p.637-645, 1963.

KIJTAWORNRAT, A.; PANYASING Y.; DEL RIO C.; HAMLIN R.L. Assessment of ECG interval and restitution parameters in the canine model of short QT syndrome, Journal of Pharmacological and Toxicological Methods, v.61, n.3, p.231-237, 2010.

KIK, M.J.L.; MITCHELL, M.A. Reptile cardiology: A review of anatomy and physiology, diagnostic approaches and clinical disease. Seminars in Avian and Exotic Pet Medicine, v.14, n.1, p.5260, 2005.

KITTLESON, M.D.; KIENLE, R.D. Small animal cardiovascular medicine, Mosby Inc., Saint Louis, 1998, Cap. 2, p. 72-94.

LIU, C.B.; LI, R.D. Electrocardiogram and heart rate in response to temperature acclimatation in three representative vertebrates. Comparative Biochemistry and Physiology A, v.142, p.416421, 2005.

MCDONALD, H.S.; HEATH, J.E. Electrocardiographic observations on the tuatara, Sphenodon punctatus. Comparative. Biochemestry Physiology A, v.40, p.881-892, 1971. 
MULLEN, R.K. Comparative eletrocardiography of the squamata. Physiological Zoology, v.40, p.114-126, 1967.

RISHER, J.F.; CLAUSSEN, D.L. The effects of cold acclimatation on electrocardiogram parameters in five species of turtles. Comparative Biochemistry and Physiology A, v.87, n.1, p.73-80, 1987.

SECOR, S.M. Specific dynamic action: a review of postprandial metabolic response. Journal of Comparative Physiology B Biochemical Systemic and Environmental Physiology, v.179, n.1, p.1-56, 2009.

SHOEMAKER, N.J.; ZANDVLIET, M.M.J.M. Electrocardiograms in selected species. Seminars in Avian and Exotic Pet Medicine, v.14, n.1, p.26-33, 2005.

TERREL, G., JONES, H., KING, R.R. Characterization of the electrocardiogram of the American Alligatot (Alligator mississippiensis). Journal of Zoo and Wildlife Medicine, v. 25, n.1, p. 40-47, 1994.

TILLEY, L.P.; GOODWIN, J.K. Manual of canine and feline cardiology, 3.ed., 2001, Cap.3, p.4371.

VALENTINUZI, M.E.; HOFF, H.E.; GEDES, L.A. Electrocardiogram of the snake: effect of the location of the electrodes and cardiac vectors. Journal of Eletrocardiology, v.2, p.245-252, 1969a.

VALENTINUZI, M.E.; HOFF, H.E.; GEDES, L.A. Electrocardiogram of the snake: intervals and duration. Journal of Eletrocardiology, v.2, p.343-352, $1969 \mathrm{~b}$. 